Www.jmscr.igmpublication.org

Impact Factor (SJIF): 6.379

Index Copernicus Value: 79.54

ISSN (e)-2347-176x ISSN (p) 2455-0450

crossrefDOI: https://dx.doi.org/10.18535/jmscr/v6i11.16

Journal Of Medical Science And Clinical Research

IGM Publication

An Official Publication of IGM Publication

\title{
A Prospective Cohort Study on the Infections Associated with Indwelling Medical Devices in a Tertiary Care Hospital in South India
}

\author{
Authors \\ Dr T.S Sushitha ${ }^{1}$, Prof Dr K.V Suseela ${ }^{2}$ \\ ${ }^{1}$ Assistant Professor, Department of Microbiology, Government Medical College Kozhikode, Kerala, \\ India-673008 \\ ${ }^{2}$ Professor and HOD, Department of Microbiology, Amala Institute of Medical Sciences Thrissur, Kerala, \\ India \\ Corresponding Author \\ Prof Dr K.V Suseela
}

Professor and HOD, Department of Microbiology Amala Institute of Medical Sciences Thrissur, Kerala, India, Mobile-8606398463

The study conducted in Department of Microbiology, Jubilee Mission Medical College and Research

Institute Thrissur, Kerala

\begin{abstract}
Devices associated infections (DAIs) have major impact on patient morbidity. This study was aimed to evaluate the active prospective surveillance to measure the prevalence of three common DAIs like catheter associated urinary tract infection (CAUTI), ventilator-associated pneumonia (VAP) and intra vascular catheter related bloodstream infection (IV-CRBSI) in different ICUs/wards in a tertiary care centre. Urine was collected by aspiration of the catheter in CAUTI, endotracheal aspirate in VAP, catheter tips and blood samples in IVCRBSI were collected and processed as per the standard microbiological techniques. Descriptive analysis of the data was done and DAI rate was expressed as number of DAI per 1000 device days. The overall infection rate for CAUTI, VAP, IV-CRBSI were found to be 7.03, 34.84 and 0.47 per 1000 device days, respectively. Major organisms isolated were Escherichia coli, Acinetobacter baumannii, Klebsiella pneumoniae, and Pseudomonas aeruginosa,. Most of them were multi drug resistant. High rates of DAIs and antimicrobial resistance require strengthening the infection control, instituting surveillance systems, and implementing evidence-based preventive strategies.
\end{abstract}

Keywords: Devices associated infections, multi drug resistant, urinary tract infection, ventilatorassociated pneumonia.

\section{Introduction}

The development in technology has allowed the use of wider and newer varieties of medical devices in the treatment and diagnosis of various diseases to improve the health or to save the lives of patients. The number of intravascular catheters, urinary catheters, endotracheal tubes and other temporary devices inserted each year probably ranges in millions. The variety of available devices and the frequency with which they are implanted will be undoubtedly increasing in the coming years. The development in the field of Geriatrics and consistently growing number of inserted devices are likely to escalate the 
occurrence of medical devices related infections. Medical devices are associated with definite risk of bacterial and fungal infection ${ }^{(1)}$. The consequences of device related infections can be disastrous which include potentially life threatening systemic infections. Furthermore, infections of devices may result in device malfunction that hence may require device removal, often complicated by tissue destruction $^{(2)}$. Surveillance of hospital acquired infections helps in determining the prevalence, risk factors and in planning the preventive strategies $^{(3-4)}$. The present study targets on three common Device associated infections (DAIs) such as Catheter associated urinary tract infections (CAUTI), Ventilator -associated pneumonia (VAP) and Intravenous catheter-related bloodstream infections (IV-CRBSI). The knowledge of prevalence of these infections, their etiological agents and antibiotic susceptibility pattern may direct the optimum antibiotic treatment that can be installed. This study aims to measure the prevalence of three common DAIs like catheter associated urinary tract infection (CAUTI), ventilator-associated pneumonia (VAP) and intra vascular catheter related bloodstream infection (IV-CRBSI) in different ICUs/wards.

\section{Methodology}

This prospective cohort study was performed over a period of one year in a 1500 bedded tertiary hospital with the approval from the Research and Ethical committees of the institution and informed consent from each patient's next of kin. The study targeted at three common DAIs like CAUTI, VAP, and IV-CRBSI. Patients with Foleys catheter in situ of duration of >3days in neurology, urology wards and neurology intensive care unit (NICU) and patients kept on mechanical ventilation for more than 48 hours in the neonatal ICU (NNICU) and Neurosurgery ICU (NSICU) were selected for the study. In patients presented with clinical symptoms and signs of septicemia after venous and arterial catheterization blood samples were collected for culture. Out patients, patients with devices of short duration i.e. <48 hours and patients not willing to enroll were excluded.

\section{Subjects and sample collection}

Along with the patient's demographic details such as name, age and sex, the details of date of hospital admission, diagnosis, type of devices, time and date of device insertion were collected. Device days were recorded every day. Physicians' notes and investigation reports were observed for any signs of infection. If infection was suspected, the appropriate clinical sample for the type of device was collected.

Urine- Two ml was collected by puncturing the catheter tubing with a needle and syringe after cleaning with $70 \%$ ethyl alcohol. Samples were transported without delay.

VAP- Endotracheal aspirate was collected by using a sterile $12 \mathrm{G}$ endotracheal suction catheter tube ${ }^{(5)}$. Suction catheter was connected to the suction pump and passed through endotracheal tube for a distance of approximately $25 \mathrm{~cm}$. Samples were transported immediately.

IV-CRBSI-Catheter tips were collected from all the patients at the removal of catheter. Catheter was removed with sterile forceps after cleaning the skin with $70 \%$ alcohol. The terminal 2-3 inches of the tip was cut with sterile scissors and transported to the laboratory as soon as possible ${ }^{(6,7)}$.

Blood samples from peripheral veins were collected from the patients with clinical evidence of sepsis. $1 \mathrm{ml}$ of blood in pediatric patients and 3$4 \mathrm{ml}$ in adult patients were collected with aseptic precautions in BAC T/ALERT culture bottles.

\section{Sample processing}

Urine- samples were examined by macroscopy as clear/turbid/blood stained. By direct microscopy the finding of $\geq 3$ leucocytes per HPF of unspun urine was considered as significant ${ }^{(8)}$.

Semi quantitative culture was done on sheep blood agar (SBA) and MacConkey agar (MA) and the plates were incubated aerobically at $37^{\circ} \mathrm{C}$ for 18-24 hrs. 
Endotracheal aspirate- Direct microscopy was done by smear preparation \& Gram staining with the purulent portion of the samples. Semi quantitative culture method by using standard calibrated loop was followed after homogenization of the sample by vortexing for 1 minute. Inoculation was performed onto SBA, MA and Chocolate agar media. The plates were incubated at $37^{\circ} \mathrm{C}$ aerobically for $18-24 \mathrm{hrs}$.

Catheter Tip-Semi quantitative method used by Maki et al (Roll over plate method) was followed for culture ${ }^{(6,7)}$. The tip was rolled back and forth across the entire surface of media like SBA and MA using sterile forceps and exerting slight downward pressure and incubated at $37^{\circ} \mathrm{C}$ aerobically for 18-24 hrs.

Blood sample- Blood culture was performed by automated method. BAC-T /ALERT bottles were loaded in the equipment. Sub culture was done on SBA and BA and incubated at $37^{\circ} \mathrm{C}$ for $18-24 \mathrm{hrs}$ from the samples with alert.

All isolates were subjected to identification tests by conventional and automation (vitek 2). Antibiotic susceptibilities were determined according to CLSI guidelines. For data collection and analysis, two important parameters were considered.

They were:

\section{Denominator data}

This included all patients exposed to the particular device during surveillance period. A total number of device days were thus calculated.

\section{Numerator data}

This included number of patients who developed bacteriologically confirmed infection on a particular device as per standard definitions. Device - associated infection rate was expressed as the number of DAIs per 1000 device days, as calculated by dividing the number of persons developing device-associated infection by the total number of device days and multiplied by 1000 .

\section{Results}

One hundred and fifty urine samples were collected from neurology ICU and wards and urology wards (Table 1). Of these 36 samples yielded significant growth (24\%). The predominant isolate was Escherichia coli followed by Candida species. Pseudomonas aeruginosa was the third predominant. Other organisms were Klebsiella pneumoniae, Enterococcus fecalis (Fig1). Among the candida isolates Candida albicans was the commonest followed by Candida tropicalis.

A total of 98 endotracheal aspiration samples were collected from neurosurgery and neonatal ICUs (Table 2). 30 samples grew significant isolates $(30.6 \%)$. The major pathogens isolated from these samples were Gram negative bacilli. Acinetobacter baumannii was found to be predominating (31\%) followed by Klebsiella pneumonia (25\%). Pseudomonas aeruginosa, Escherichia coli and MRSA were other pathogens isolated (Fig 2).

Two hundred and forty intravenous cannula tips sere collected for microbiological study (Table 3). Organisms found to be colonizing were Coagulase negative staphylococcus (32\%) followed by Candida spp and K. pneumonia and Enterococcus fecalis (Fig 3). The only isolate causing blood stream infection in the present study was $K$. pneumonia, resulting an infection rate of $0.41 \%$. A very high resistance was observed in our syudy. $84.21 \%$ of the total Gram negative isolates of the present study were found to be extended spectrum betalactamase producers (Table 4). All Gram positive isolates were susceptible to Vancomycin \& Linezolid. $75 \%$ S.aureus isolates were Methicillin resistant. Ventillator associated pneumonia (30.6) was the commonest DAI followed by Catheter associated urinary tract infection (24\%) and IV catheter related blood steam infection $(0.41 \%)$ in our hospital (Table 5). 


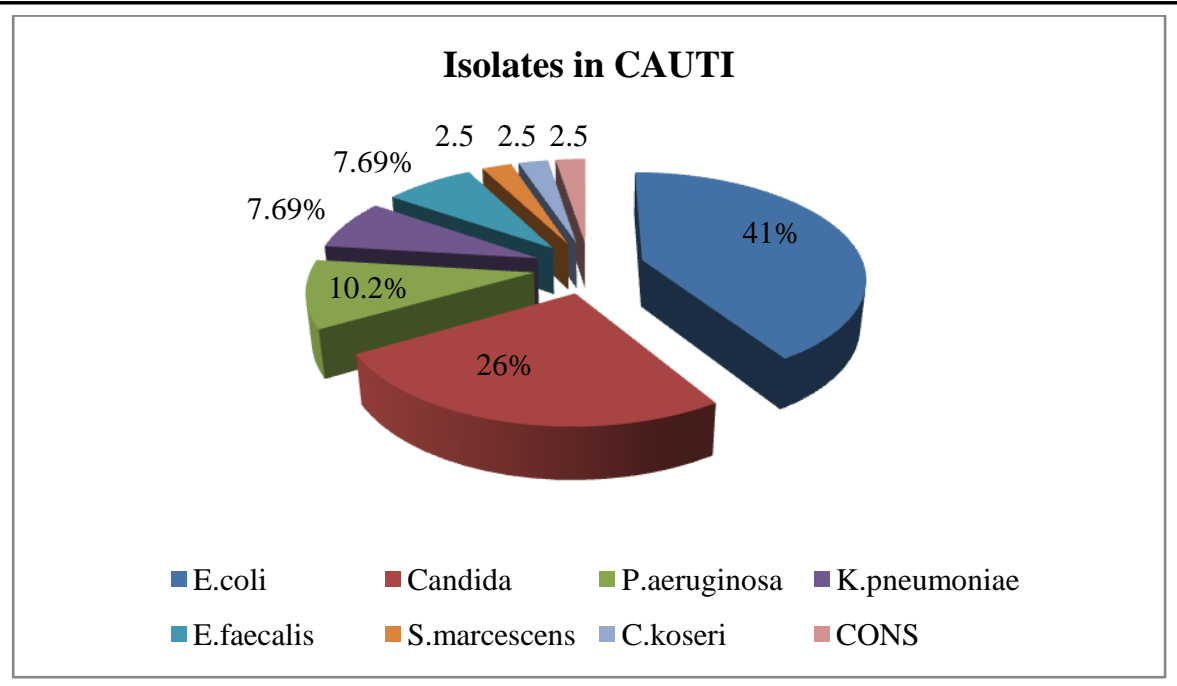

Fig 1. Distribution of isolates in CAUTI

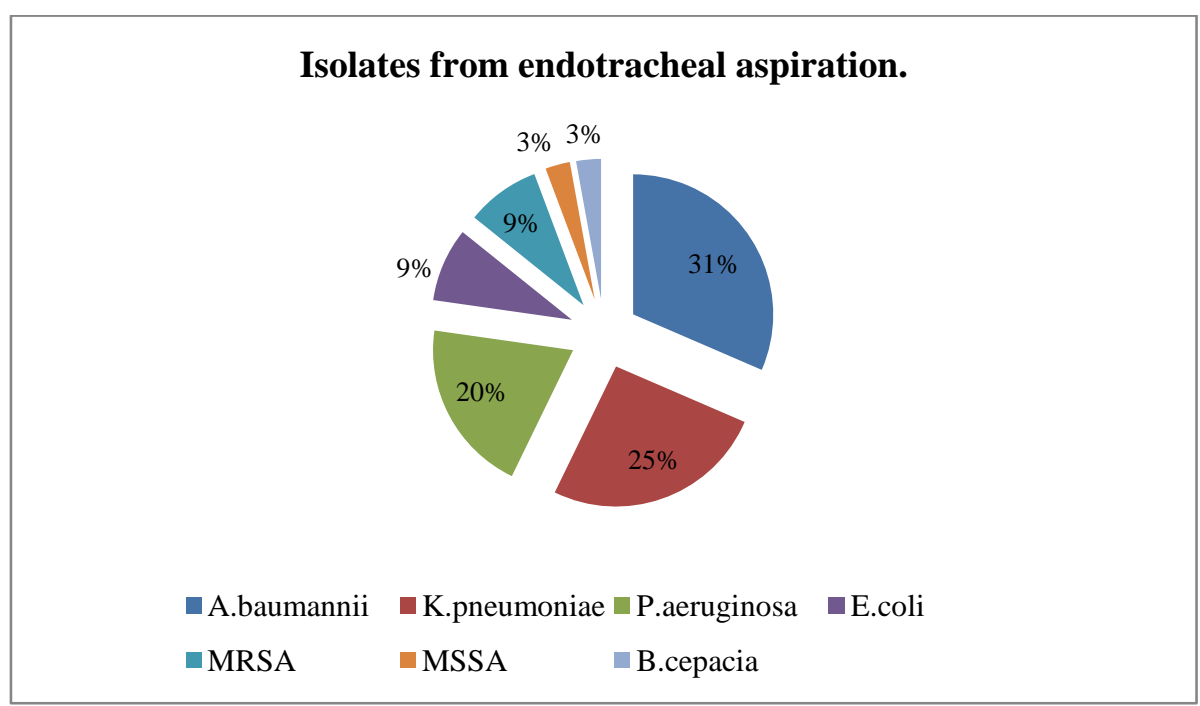

Figure 2 Distribution of bacteria in VAP

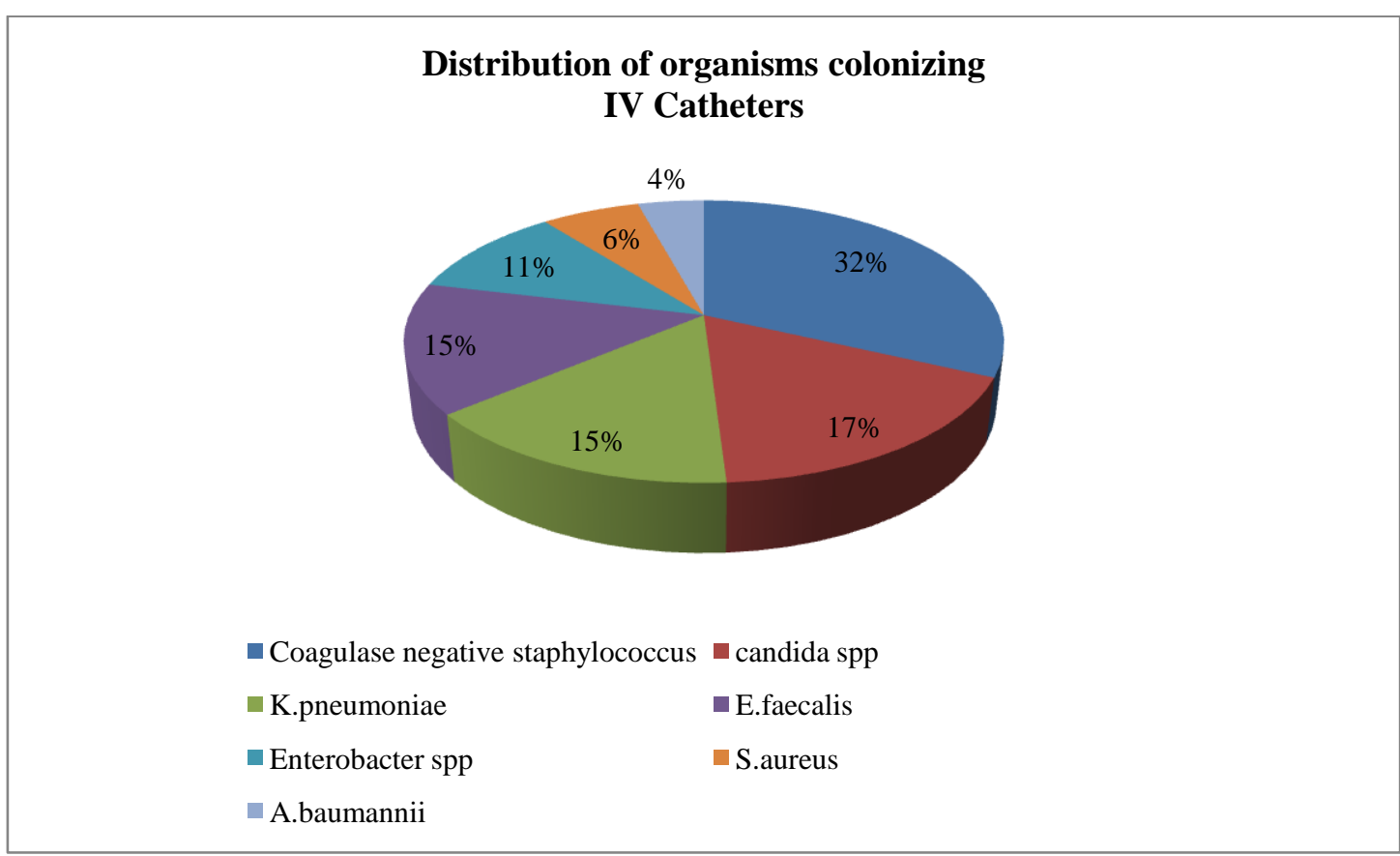

Fig 3. Distribution of bacteria in IV-CRBSI 
Table 1 Distribution of patients on indwelling urinary catheter.

\begin{tabular}{|l|c|}
\hline ICU/Ward & No of patients studied \\
\hline Neurology ward & 76 \\
\hline Neurology ICU & 44 \\
\hline Urology ward & 30 \\
\hline Total & 150 \\
\hline
\end{tabular}

Table 2: Distribution of patients in Ventilator

\begin{tabular}{|l|c|}
\hline ICU & No of patients studied \\
\hline NSICU & 60 \\
\hline NNICU & 38 \\
\hline Total & 98 \\
\hline
\end{tabular}

Table 3 Distribution of patients in IV-CRBSI

\begin{tabular}{|l|c|}
\hline ICU & No of patients studied \\
\hline Neonatal ICU (NNICU) & 116 \\
\hline Multiple critical care unit (MCCU) & 37 \\
\hline Medical ICU (MICU) & 24 \\
\hline Neurology ICU (NICU) & 22 \\
\hline Pediatric ICU (PICU) & 16 \\
\hline Neurosurgery ICU & 25 \\
\hline Total & 240 \\
\hline
\end{tabular}

Table 4 Antibiotic susceptibility pattern of ESBL producing pathogens from the total Gram negative isolates

\begin{tabular}{|l|c|c|c|}
\hline \multirow{2}{*}{ No of Isolates } & \multicolumn{3}{|c|}{ Susceptible to (percentage) } \\
\cline { 2 - 4 } & IPM & TGC & CL \\
\hline E.coli $(\mathrm{n}=16)$ & $11(68.75)$ & $15(93.75)$ & $16(\mathrm{I} 00)$ \\
\hline A.baumannii $(\mathrm{n}=11)$ & $4(36.36)$ & $11(\mathrm{I} 00)$ & $11(\mathrm{I} 00)$ \\
\hline K.pneumoniae $(\mathrm{n}=12)$ & $10(83.3)$ & $12(\mathrm{I} 00)$ & $12(\mathrm{I} 00)$ \\
\hline P.aeruginosa $(\mathrm{n}=8)$ & $7(87.5)$ & - & $8(\mathrm{I} 00)$ \\
\hline S.marcescens $(\mathrm{n}=1)$ & $1(\mathrm{I} 00)$ & $1(\mathrm{I} 00)$ & $1(\mathrm{I} 00)$ \\
\hline
\end{tabular}

IPM -Imipenem, TGC- Tigecycline, CL- Colistin

Table 5: Prevalence of dais

\begin{tabular}{|l|c|c|c|}
\hline $\begin{array}{l}\text { Type of } \\
\text { infection(DAIs) }\end{array}$ & $\begin{array}{c}\text { Total no of cases } \\
\text { studied }\end{array}$ & Prevalence (\%) & $\begin{array}{c}\text { Prevalence per 1000 } \\
\text { device days }\end{array}$ \\
\hline CAUTI & 150 & 24 & 7.03 \\
\hline VAP & 98 & 30.6 & 34.84 \\
\hline IV-CRBSI & 240 & 0.41 & 0.47 \\
\hline
\end{tabular}

\section{Discussion}

In the present study, a prospective surveillance of three common DAIs like catheter-associated urinary tract infection (CA-UTI), ventilatorassociated pneumonia (VAP) and IV-catheterrelated bloodstream infections (IV-CRBSI) to determine the prevalence, etiology and antibiotic susceptibility pattern of the isolates was carried out between June 2012 and May 2013, in different wards/ICUs in a tertiary care centre in Kerala.

A total number of 150 catheterized patients were studied. Only 36 cases of CAUTI were confirmed microbiologically. The overall prevalence rate of
CAUTI was $24 \%$ or in other words 7.03 per 1000 catheter days in our study, which is high compared to earlier studies ${ }^{(9,10)}$. An Indian study by Singhai et al. reported high prevalence rate of $26.6 \%$ similar to the present study ${ }^{(11)}$. Rates will vary in studies according to how long the patients have been catheterized and how often urine cultures are performed. Similar to earlier studies, ${ }^{(9-11)}$ in the present study, we found the Gram negative bacilli were the major isolates comprising Enterobacteriaceae (53.84\%) and Nonfermenters (10.25\%) . E.coli was predominant (41\%) which was consistent to the previous 
studies $^{(9-11)}$ followed by Candida spp, $P$. aeruginosa, K. pneumonia and E. faecalis. Manish et al. had reported that $100 \%$ of E. coli isolates were susceptible to Imipenem, $82.46 \%$ to Nitrofurantoin and $87.7 \%$ to Amikacin. In our study, E. coli were found to be susceptible to Amikacin (68.75\%), Imipenem (68.75\%), Cefoperazone + Sulbactum (62.5\%), Nitrofurantoin and Piperacillin+Tazobactam (56.25\% each).

Increasing antibiotic resistance is a major clinical problem faced by clinicians in treating infections by these microorganisms.. Similar to other reports, (11-13) in the present study also majority of the uropathogens in the catherized patients were resistant to Ampicillin, Ciprofloxacin, Cotrimoxazole and Gentamicin. Among the $E$. coli isolates 81.25 were extended spectrum beta lactamase (ESBL) producers. Dugal et al. ${ }^{13}$ has reported $70.9 \%$ ESBL producing E. coli isolates in catheterized patients. The rate of antibiotic resistance of the isolates in our study was found to be high compared to other Indian studies. ${ }^{(11-13)}$

VAP is an important DAI as a result of mechanical ventilation in the available studies ${ }^{(14-}$ 17) 98 patients kept on ventilation for $>48 \mathrm{hrs}$ were included in the study. Out of the clinically suspected cases, 30 patients were confirmed microbiologically ensuing an infection rate of $30.6 \%$ or in other words 34.84 infections per 1000 ventilator days. The rate of VAP in the present study is high similar to other Indian studies ${ }^{(14-16)}$. The causative organisms vary with the patients demographics in the ICU, the method of diagnosis, the duration of hospital stay and the institutional antimicrobial policies. VAP may be caused by a wide spectrum of bacterial pathogens. In the present study, Gram negative bacteria were the most common pathogens of VAP, as observed in other studies. ${ }^{(14-17)}$ Earlier studies ${ }^{(15,16)}$ had shown that Pseudomonas and Klebsiella were the common organisms. In contrast to previous studies in the present study, Acinetobacter baumanniiwas found to be the most common organism causing VAP followed by $K$. pneumoniae, P. aeruginosa, E. coli, B. cepacia and $S$. aureus. Similar bacteriological profile was found in recent studies by Chawla et $\mathrm{al}^{(18)}$ and Ranjit et $\mathrm{al}^{(17)}$.

Chawla et $\mathrm{al}^{(18)}$ was demonstrated that Acinetobacter spp was found to be emerging in several countries, notably Malaysia, Thailand, Pakistan and India . Recent studies have shown that the increasing incidence of multidrug resistant pathogens are seen among the pathogens with VAP $^{(14-17)}$ Although the Acinetobacter species is less virulent than Pseudomonas, they are becoming more and more resistant to the commonly used antimicrobial agents. So in coming future infections by Acinetobacter may be a major problem in the similar clinical settings. Of the isolates in VAP in the present study,

A. baumaniiwas found to be the most resistant one. None of the Acinetobacter isolates were found to be susceptible to Ampicillin (AMP), Gentamicin $(\mathrm{G})$, third generation cephalosporins, ciprofloxacin(CIP) and piperacillin +tazobactum (PT) combinations in the present study. 36.3\% were found to be susceptible to Imipenem (IPM), $18.1 \%$ to Amikacin (AK) \& Cefoperazone+ Sulbactum (CM). K. pneumoniae isolated in this study was susceptible to $\mathrm{CM}$ (44.4\%), AK (66.6\%), PT (33.3\%) and IPM (88.8\%). Pseudomonas aeruginosa was found to be susceptible to PT (71.4\%), CM (57.1\%), AK $(28.5 \%)$ and IPM $(85.7 \%)$. All the Gram negative pathogens were susceptible to Colistin and Tigecycline.

In the present study all $S$. aureus isolates were susceptible to Vancomycin and Linezolid. The rate of antibiotic resistance among the isolates in our study is high, similar to other studies conducted in various institutions in India ${ }^{(14-16)}$. For IV- CRBSI surveillance, IV catheter tips of 240 patients were studied. CRBSI was confirmed only in one case, with an infection rate of $0.41 \%$ or in other words 0.47 infections per 1000 device days. The infection rate in present study is quite low in comparison to another Indian study ${ }^{(19)}$. In one recent study Singh et al has reported a rate of 0.48 
per 1000 device days similar to the present study $^{(9)}$.

Colonization rate of IV catheter tips in the current study is low (19.5\%) in comparison to the previous study ${ }^{(19)}$. The isolate obtained in CRBSI in the present study was $K$. pneumoniae. Gram positive organisms were the most common cause of blood stream infections in most of the previous studies $^{(19,20)}$. Among the colonization associated organisms, Coagulase negative staphylococcus were the major isolates followed by Candida spp and $K$. pneumoniae similar to other Indian study (20). The organisms commonly found in HAIs are nosocomial pathogens prevalent in the hospital environment. The organisms isolated in the present study were multi drug resistant $K$. pneumoniae, E. coli, A. baumannii, P. aeruginosa, $S$. aureus, E. faecalis and CONS; similar to the findings in other studies ${ }^{(9-10,14-17)}$.

The DAI rates as evident in literature are highly variable, depending upon a number of factors such as type of patients admitted, prescribing habits of the clinicians, antibiotic policy and infection control programs of the institute. Surveillance of health care associated infections is important to understand the nature and extent of the problem. The present study proves that the DAIs were caused mainly by drug resistant bacteria. The varying resistance pattern of organisms isolated in our study, emphasizes the importance of studying the pattern of infection in every setting. Each institution and each ICU must have an antibiotic policy of its own which should be frequently revised by active surveillance programme.

\section{Conclusion}

Prevalence of CA-UTI was $24 \%$ or in other words 7.03 infections per 1000 catheter days. E.coli $(41 \%)$ was the major isolate followed by Candida species (26\%), P. aeruginosa (10.2\%), $K$. pneumoniae (7.69\%), E. faecalis (7.69\%)). Candida albicans $(60 \%)$ was the common fungal isolate followed by C.tropicalis (30\%). Infection rate of VAP in our institution was found to be $30.6 \%$ or 34.84 per 1000 ventilator days.
A.baumannii (31\%)was the predominant isolate followed by $K$. pneumoniae (25\%), P.aeruginosa (20\%), E.coli (9\%), MRSA(9\%), MSSA(3\%), B. cepacia $(3 \%)$ in VAP. CRBSI was confirmed only in one case, with an infection rate of $0.41 \%$ or in other words 0.47 per 1000 device days. Colonization rate of IV catheters in the current study was $19.5 \%$. Isolate obtained in CRBSI was K.pneumoniae which was susceptible only to Tigecycline and Colistin. In this study major isolates were Gram negative bacilli (76\%). Among the total Gram negative isolates, $84.21 \%$ were found to be ESBL producers.

\section{Acknowledgement}

Authors gratefully acknowledge the valuable help of Dr Ajith TA., Professor, Department of Biochemistry, Amala Institute of Medical Sciences, Thrissur, Kerala, India during the preparation of the manuscript.

Funding: No funding sources

Conflict of interest: None declared

Ethical Approval: The study was approved by the Institutional Ethics Committee

\section{References}

1. Richards MJ, Edwards JR, Culver DH, Gaynes RP. Nosocomial infections in medical intensive care units in the United States. National Nosocomial Infections Surveillance System. Crit Care Med 1999;27:887-92.

2. Darouiche RO. Device-Associated Infections: A Macroproblem that Starts with Microadherence. Clin Infect Dis 2001;33(9):1567-1572.

3. Gasink LB, Lautenbach E. Prevention and treatment of healthcare acquired infections. Med Clin North Am2008; 92:295-313.

4. Hedrick TL, Sawyer RG. Health-care associated infections and prevention. Surg Clin North Am 2005;85:1137-52.

5. Chandrakanth C, Anushree, Vinod A. Incidence of ventilator associated 
Pneumonia. Int J Med Clin Res 2010;1(2): 11-13.

6. Safdar N, Fine JP, Maki DG. Metaanalysis: methods for diagnosing intravascular device-related bloodstream infection. Ann Intern Med 2005;142:451466.

7. Maki DG, Weise CE, Sarafin HW. A semi quantitative culture method foridentifying intravenous catheter-related infections $N$ Engl J Med1977;296:1305-1309.

8. Horan TC, Andrus M, Dudeck MA. CDC/NHSN surveillance definitionof health care-associated infection and criteria for specific types of infectionsin the acute care setting. Am $J$ Infect Control2008;36:309-332.

9. Singh S, Pandya Y, Patel R, Paliwal M, Wilson A, Trivedi S. Surveillance of device associated infections at a teaching hospital in rural Gujarat - India. Indian $J$ Med Microbiol 2010;28(4):342-7.

10. Vonberg RP, Behnke M, Geffers C, Sohr D, Ruden H, Dettenkofer M, et al. Device associated infection rates for non-intensive care unit patients. Infect Control Hosp Epidemiol2006;27:357-61.

11. Singhai M, Malik A, Shahid M, Malik MA, Goyal R. A Study on device-Related Infections with Special Reference to Biofilm Production and Antibiotic Resistance. J Glob Infect Dis 2012;4(4): 193-198.

12. Manish N, Tankhiwale NS. Study of microbial flora in patients with indwelling catheter. Int J Cur Res Rev 2013;5(12):57-60.

13. Dugal S, Purohit H. Antimicrobial susceptibility profile and detection of extended spectrum beta-lactamase production by gram negative uropathogens. Int J Pharm Pharmaceutic Sci 2013;5(4) 434-438.
14. Payal MP, Tanuja JB, Sandeep N, Neelam PN. A study on ventilator associated pneumonia in pediatric age group in a tertiary care hospital, Vadodara. Natl J Med Res 2012; 2(3): 318-321.

15. Tripathi S, Jain A, Kohli N. Study Of Ventilator Associated Pneumonia In Neonatal Intensive Care Unit: Charecterisitcs, Risk Factors and Outcome. Internel J Med Update 2010;5(1):12-9.

16. Gupta A, Agrawal A, Mehrotra S, Singh A, Malik S, Khanna A. Incidence, risk stratification, antibiogram of pathogens isolated and clinical outcome of ventilator associated pneumonia. Indian J Crit Care Med 2011; 15(27): 96-101.

17. Ranjit S, Bhattarai B. Incidence and risk factors for ventilator-associated pneumonia in Kathmandu University Hospital. Kathmandu Univ Med J 2011;33 (1)28-31.

18. Chawla R. Epidemiology, aetiology and diagnosis of hospital acquired pneumonia and ventilator associated pneumonia in Asian countries. Am J Infect Control 2008; 36 (4): 93- 100.

19. Chopdekar K, Chande C, Chavan S, Veer $\mathrm{P}$, Wabale $\mathrm{V}$, Vishwakarma $\mathrm{K}$, et al. Central venous catheter-related blood stream infection rate in critical care units in a tertiary care, teaching hospital in Mumbai. Indian J Med Microbiol 2011; 29: 169-171.

20. Parameswaran R, Sherchan JB, Varma MD, Mukhopadhyay C, Vidyasagar S. Intravascular catheter-related infections in an Indian tertiary care hospital. J Infect Dev Ctries2011; 5(6):452-458. 